\title{
Insulin use and fracture risk in patients with type 2 diabetes: A meta-analysis of 138,690 patients
}

\author{
YUXIAN ZHANG $^{1 *}$, QIUYING CHEN ${ }^{2,3 *}$, YUYING LIANG $^{4}$, YUHAO DONG $^{5}$, \\ XIAOKAI MO ${ }^{5}$, $\mathrm{LU} \mathrm{ZHANG}^{5}$ and BIN ZHANG $^{2,3}$ \\ ${ }^{1}$ Department of Orthopedics, Zhujiang Hospital, Southern Medical University, Guangzhou, Guangdong 510000; \\ ${ }^{2}$ Department of Radiology, The First Affiliated Hospital, Jinan University, Guangzhou, Guangdong 510627; \\ ${ }^{3}$ Department of Imaging and Nuclear Medicine, Graduate College, Jinan University; ${ }^{4}$ Department of Radiology, \\ The First Affiliated Hospital, Guangdong Pharmaceutical University; ${ }^{5}$ Department of Imaging and Nuclear \\ Medicine, Graduate College, Southern Medical University, Guangzhou, Guangdong 510000, P.R. China
}

Received October 15, 2018; Accepted February 22, 2019

DOI: $10.3892 /$ etm.2019.7461

\begin{abstract}
Patients with type 2 diabetes mellitus (T2DM) have been reported to have an enhanced risk of bone fracture, however the association between insulin treatment and risk of fracture in patients with T2DM remains to be fully elucidated. The aim of the present meta-analysis was to examine the possible association between insulin treatment and risk of fracture in patients with T2DM. Relevant studies published prior to and including April 2018 were identified by literature searches in PubMed, Embase and Cochrane Library databases. A meta-analysis was performed, which included relevant trials of patients with T2DM comparing insulin to oral anti-diabetic drugs. The combined effect was expressed as a pooled risk ratio (RR) with $95 \%$ confidence interval (CI), using a random-effects model. Subgroup analysis was performed to consolidate the results. A total of 7 studies comprising 138,690 patients were eligible for inclusion in the present meta-analysis. After exclusion of one study that introduced major heterogeneity, treatment with, insulin was associated with a significantly increased risk of fracture among patients with T2DM ( $\mathrm{RR}=1.24$, 95\% CI, 1.07-1.44; $\mathrm{P}=0.004)$. Subgroup analysis by sex indicated that the $\mathrm{RR}$ for men was $1.04(95 \% \mathrm{CI}, 0.76-1.44, \mathrm{P}=0.801)$ and that for women was 1.22 (95\% CI, 0.92-1.62, P=0.175). Subgroup analysis by fracture site indicated that the RR for hip was 1.18 (95\% CI, 0.83-1.68, $\mathrm{P}=0.363$ ), that for vertebrae was 1.28 (95\% CI, 0.90-1.81, $\mathrm{P}=0.169)$ and that for non-vertebrae was 1.06 (95\% CI, 0.80-1.41, P=0.686). Subgroup analysis
\end{abstract}

Correspondence to: Dr Bin Zhang, Department of Radiology, The First Affiliated Hospital, Jinan University, 613 Huangpu West Road, Guangzhou, Guangdong 510627, P.R. China

E-mail: xld_Jane_Eyre@126.com

"Contributed equally

Key words: insulin, type 2 diabetes, fracture, meta-analysis by study design suggested that the RR for prospective and retrospective studies was 1.35 (95\% CI, 1.06-1.71, $\mathrm{P}=0.014)$ and 1.16 (95\% CI, 0.99-1.35, P=0.059), respectively. Subgroup analysis by region indicated that the RR for Europe was 1.16 (95\% CI, 0.91-1.48, $\mathrm{P}=0.220$ ), that for North America was 1.24 (95\% CI, 0.81-1.90, $\mathrm{P}=0.333$ ) and that for Asia was 1.34 (95\% CI, 0.91-1.98, P=0.141). In conclusion, treatment with insulin increased the risk of fractures among patients with T2DM compared with oral anti-diabetic drugs; however, the association was influenced by various factors, including sex, fracture site, study design and geographical region.

\section{Introduction}

Bone fracture, particularly in the hip and vertebrae, is a major cause of morbidity and mortality among patients with diabetes mellitus (DM) (1). It is evident that diabetic patients have a higher fracture risk when compared with non-diabetic patients (2-4). In contrast to type $1 \mathrm{DM}$ (T1DM), T2DM is associated with an increased body mass index (BMI) and higher bone mineral density (BMD), which is paradoxical to the increased fracture risk (5). This indicates that the BMD may not reflect bone fragility in patients with T2DM. Insulin deficiency and dysfunction, obesity and hyperinsulinemia, altered level of estrogen, leptin and adiponectin, as well as diabetes-associated complications, particularly peripheral neuropathy, orthostatic hypotension or reduced vision due to retinopathy may all be associated with an impairment of bone metabolism and increased risk of fracture (5). In addition, medications used for diabetes management may have an important role in bone metabolism and fracture risk.

The association between insulin use and fracture risk in T2DM is being increasingly recognized. Insulin induces anabolic effects on the bone, which includes the regulation of bone cell proliferation and apoptosis, and the synthesis of collagen (6). Most of the studies published to date have indicated that insulin-treated diabetes is associated with an increased risk of bone fracture $(1,2,4,7-9)$; However, the conclusions of certain studies appear to be inconsistent $(10,11)$. Kennedy et al (12) confirmed that patients taking insulin had an 
increased risk of accidents, which was most likely associated with the increased tendency of insulin-treated patients to fall during a hypoglycemic episode. To test the hypothesis that insulin use is associated with fracture risk among patients with T2DM and to determine confounding factors that may account for any association, the present meta-analysis was performed.

\section{Materials and methods}

Search strategy. PubMed, Embase and Cochrane Library databases were searched for studies published prior to and including April 2018 without any restrictions regarding country, publication type or language. Combinations of the following key words were used: [insulin (Title/Abstract)] AND [fracture (Title/Abstract)] AND [diabetes (Title/Abstract)]. As the search terms and strategy did not retrieve all relevant articles, the references listed in eligible papers were also screened to supplement the results. Details of the search strategy implemented in the current study are illustrated in Fig. 1.

Inclusion and exclusion criteria. Two authors (XKM and YYL) independently performed literature searches, preliminary screening study titles and abstracts. The full-text articles were subsequently evaluated according to the inclusion and exclusion criteria; a third author (YHD) extracted valuable data from the eligible studies to be included in the analysis. Any discrepancies were resolved through discussion. The inclusion criteria were as follows: i) The participants were diagnosed with T2DM; ii) each study used oral anti-diabetic drugs as the control arm; iii) the duration of the study was at least one year; and iv) complete fracture data for the insulin and non-insulin groups were available. Studies were excluded if: i) They compared their dataset to the general population; and ii) only reported on T1DM or did not classify the DM type.

Data synthesis and analysis. Considering the heterogeneity of the included studies, the random-effects model was used to calculate the pooled risk ratio (RR), with a $95 \%$ confidence interval (CI). Heterogeneity was evaluated using $\mathrm{I}^{2}$ statistics, with values of $>25,>50$ and $>75 \%$ representing low, moderate and high heterogeneity, respectively. A sensitivity analysis was performed to verify the robustness of the results by removing one study at a time. If there was a significant change in heterogeneity after removing a study, then this study was removed. To assess sources of heterogeneity, subgroup analyses were performed based on sex, fracture sites, region and study design. The results were regarded as statistically significant if a two-sided $\mathrm{P}<0.05$ was obtained. Statistical analyses were performed using Comprehensive Meta-Analysis version 2 (www.Meta-Analysis.com) and Stata (version 12; www.Stata.com).

\section{Results}

Studyselection.A total of 344 records were selected by searching the databases and the literature references of the articles retrieved. After 337 exclusions, 7 articles (1,3,8,13-16) were determined to be eligible for the present meta-analysis (Fig. 1). Overall, 138,690 patients were enrolled in the studies selected (3,879 insulin users and 134,811 non-insulin users); among them, 5,798 cases of fracture were reported. The publication dates for the studies ranged from 2001 to 2018 and the follow-up time ranged from 2.3 to 22.0 years. The study population mostly included middle-aged and elderly participants. Among the eligible studies, two studies exclusively included women. Fracture sites included the vertebrae, hip and other non-vertebral locations. The study locations included Asia, Europe and North America. The study design included prospective and retrospective studies. The characteristics of the seven trials are provided in Table I.

Association between insulin intake and risk of fracture in patients with T2DM. Overall, the pooled data from the seven trials evaluating the risk of fractures associated with insulin used in diabetic patients indicated that insulin did not significantly increase the risk of fracture compared to oral anti-diabetic drugs $(\mathrm{RR}=1.13,95 \% \mathrm{CI}, 0.92-1.39$; $\mathrm{P}=0.252$; Fig. 2). However, the $\mathrm{I}^{2}$ values indicated a significant heterogeneity among the trials included $\left(\mathrm{I}^{2}=84.1 \%\right)$. Considering the obvious heterogeneity, a sensitivity analysis and subgroup analysis were performed to explore the sources of heterogeneity.

Sensitivity and subgroup analyses. A sensitivity analysis was performed to verify the robustness of the results by removing one study at a time. The analysis revealed that the results changed following the exclusion of the study conducted by Pscherer et al (13) ( $\mathrm{RR}=1.24,95 \% \mathrm{CI}, 1.07-1.44 ; \mathrm{P}=0.004$; Fig. 3), with the heterogeneity markedly decreased $\left(\mathrm{I}^{2}=41.1 \%\right)$.

Subsequently, subgroup analyses by sex, fracture site, study design and geographical region were performed to identify the sources of heterogeneity. In the subgroup analysis by sex, the corresponding RR for males was 1.04 (95\% CI, 0.76-1.44, $\mathrm{P}=0.801)$ and that for females was 1.22 (95\% CI, 0.92-1.62, $\mathrm{P}=0.175)$, while that for females and males combined was 1.26 (95\% CI, 1.05-1.51, P=0.013; Fig. 4). No heterogeneity was identified in the subgroup of males $\left(\mathrm{I}^{2}=0.0 \%\right)$, whereas the female and mixed sex subgroups demonstrated moderate heterogeneity ( $\mathrm{I}^{2}=56.4$ and $55.0 \%$, respectively).

In the subgroup analysis by fracture site, the corresponding RR for hip was 1.18 (95\% CI, 0.83-1.68, $\mathrm{P}=0.363)$, for non-vertebral sites (including hip) was 1.06 (95\% CI, 0.80-1.41, $\mathrm{P}=0.686)$ and for vertebral was 1.28 (95\% CI, 0.90-1.81, $\mathrm{P}=0.169$ ) (Fig. 5). The heterogeneity in the non-vertebral (including hip), vertebral and hip subgroups were 0.0,39.6 and $66.7 \%$, respectively.

In the subgroup analysis by study design, the corresponding RR for prospective and retrospective study subgroups was 1.35 (95\% CI, 1.06-1.71, $\mathrm{P}=0.014)$ and 1.16 (95\% CI, 0.99-1.35, $\mathrm{P}=0.059$ ), respectively (Fig. 6). The heterogeneity was low in the retrospective study subgroup $\left(\mathrm{I}^{2}=5.8 \%\right)$ and moderate in the prospective study subgroup $\left(\mathrm{I}^{2}=52.4 \%\right)$.

In the subgroup analysis stratified by geographical region, the corresponding $\mathrm{RR}$ for North America was 1.24 (95\% CI, 0.81-1.90, $\mathrm{P}=0.333$ ), that for Asia was 1.34 (95\% CI, 0.91-1.98, $\mathrm{P}=0.141)$ and that for Europe was 1.16 (95\% CI, 0.91-1.48, P=0.220; Fig. 7). The heterogeneity was low in the Europe subgroup $\left(\mathrm{I}^{2}=18.0 \%\right)$, but moderate in the North America and Asia subgroups $\left(\mathrm{I}^{2}=64.7\right.$ and $58.2 \%$, respectively). 


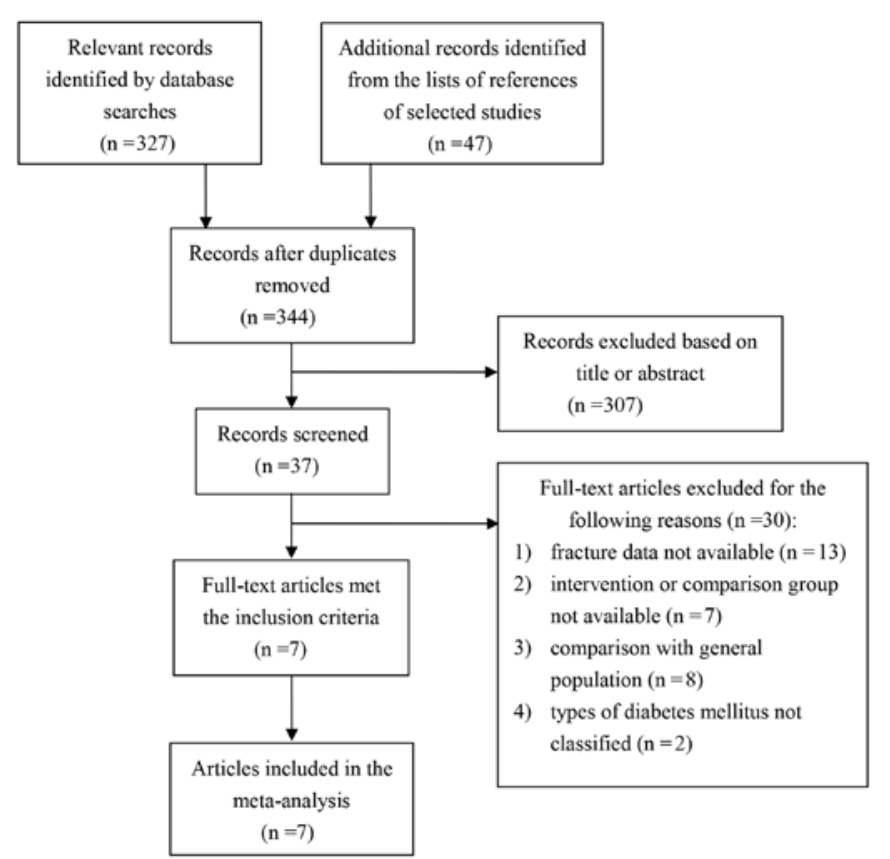

Figure 1. Flow chart for the selection of articles for inclusion in the present meta-analysis.

\section{Discussion}

Insulin has been used as one of several therapeutic options for diabetic patients for numerous years. However, the association between insulin use and fracture risk of diabetes patients remains controversial. It is important to clarify this issue, as bone fractures, particularly those of the hip and vertebrae, are a major cause of disability and mortality among elderly patients with T2DM. Therefore, a meta-analysis was performed to determine the potential association between insulin use and fractures in patients with T2DM.

After exclusion of the study by Pscherer et al (13), the corrected meta-analysis indicated that the use of insulin significantly increased the risk of fractures among patients with T2DM compared with oral antidiabetic drugs, which was consistent with the results from previously published studies $(1,2,7-9,15)$. Subgroup analyses based on sex, study design, fracture sites and geographical region were employed to detect subgroup differences. The subgroup analysis by sex indicated that insulin significantly enhanced the risk of fracture from females and males combined, while there was so significant difference between males and females and no significant association was identified for either sex separately. When the cohort was stratified according to study design, the prospective study subgroups exhibited significantly higher fracture risk associated with insulin treatment, whereas no such association was observed in the retrospective subgroups. In addition, this association was not observed in the subgroups that were stratified according to fracture site and the region.

The source of heterogeneity in a meta-analysis should be comprehensively investigated to avoid any possible adverse influences. Considerable heterogeneity was present in the initial meta-analysis, and the possible source

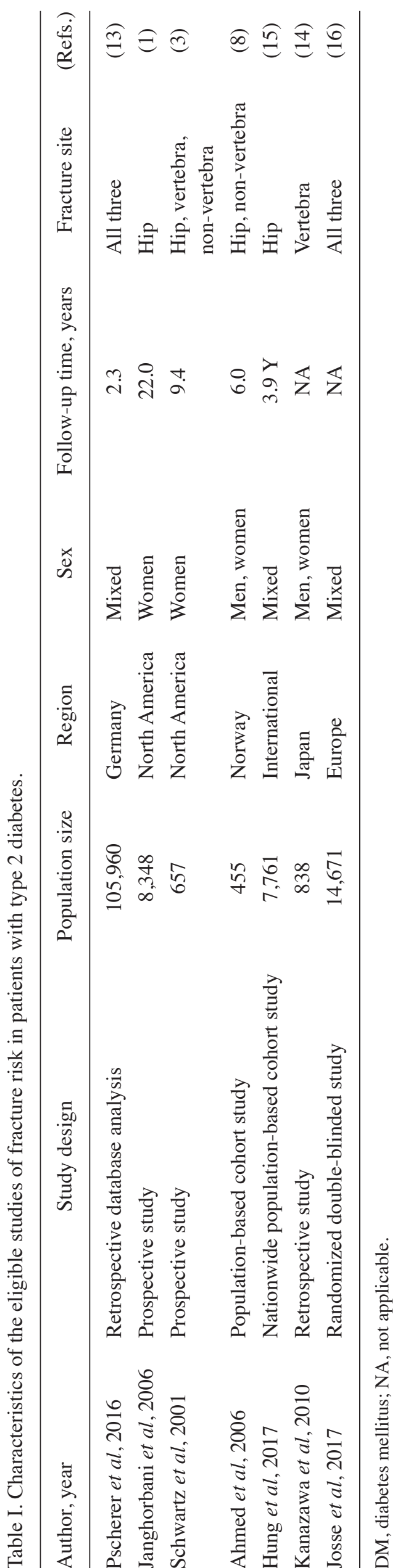




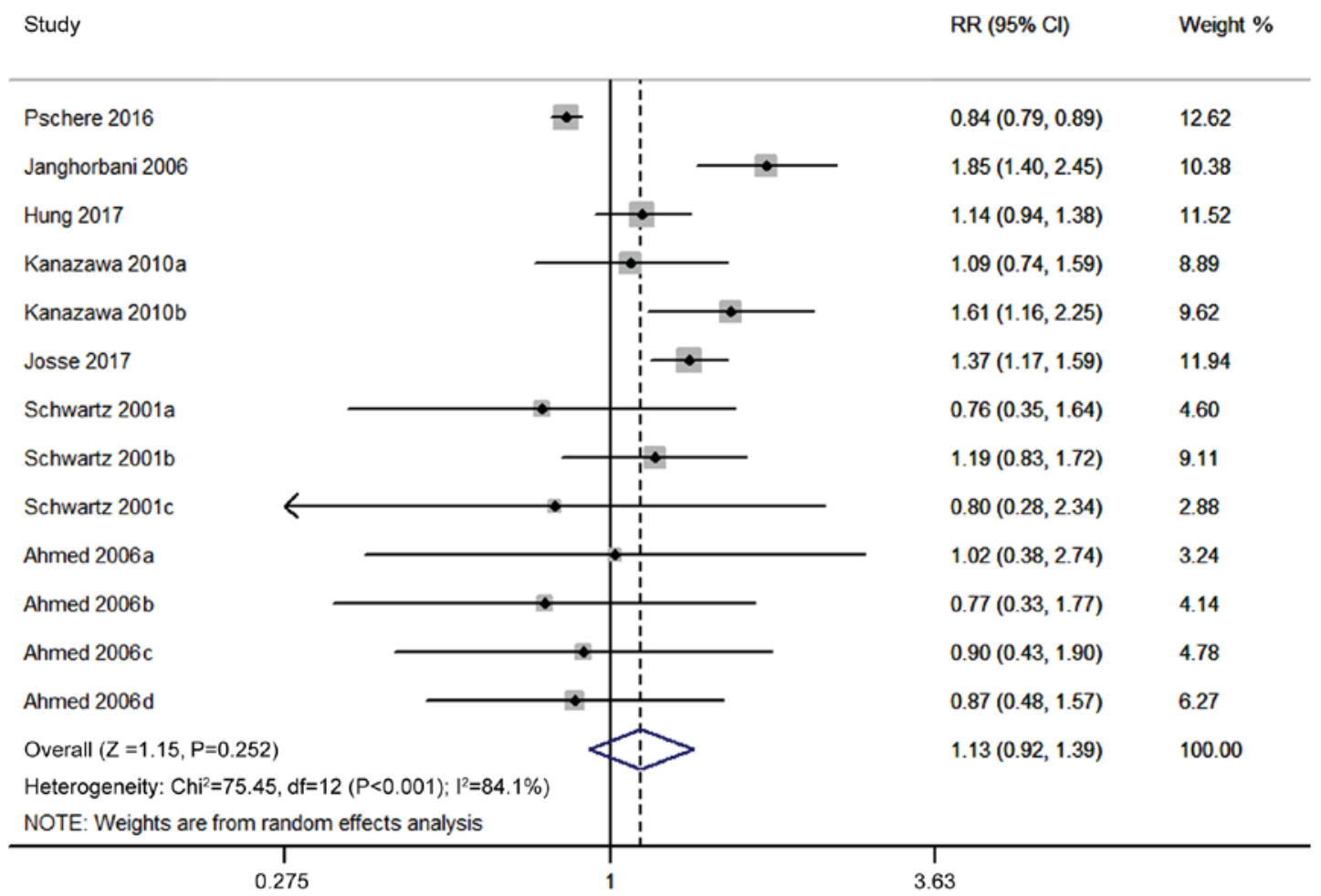

Figure 2. Forest plot comparing the risk of fracture following insulin and non-insulin treatment for patients with type 2 diabetes mellitus. The risk ratio and weight for each study are presented as solid diamond and squares, respectively. The horizontal line indicates the $95 \% \mathrm{CI}$ and the dotted line indicates the pooled risk ratio. Pooled results for all studies are presented as an unfilled diamond. CI, confidence interval.

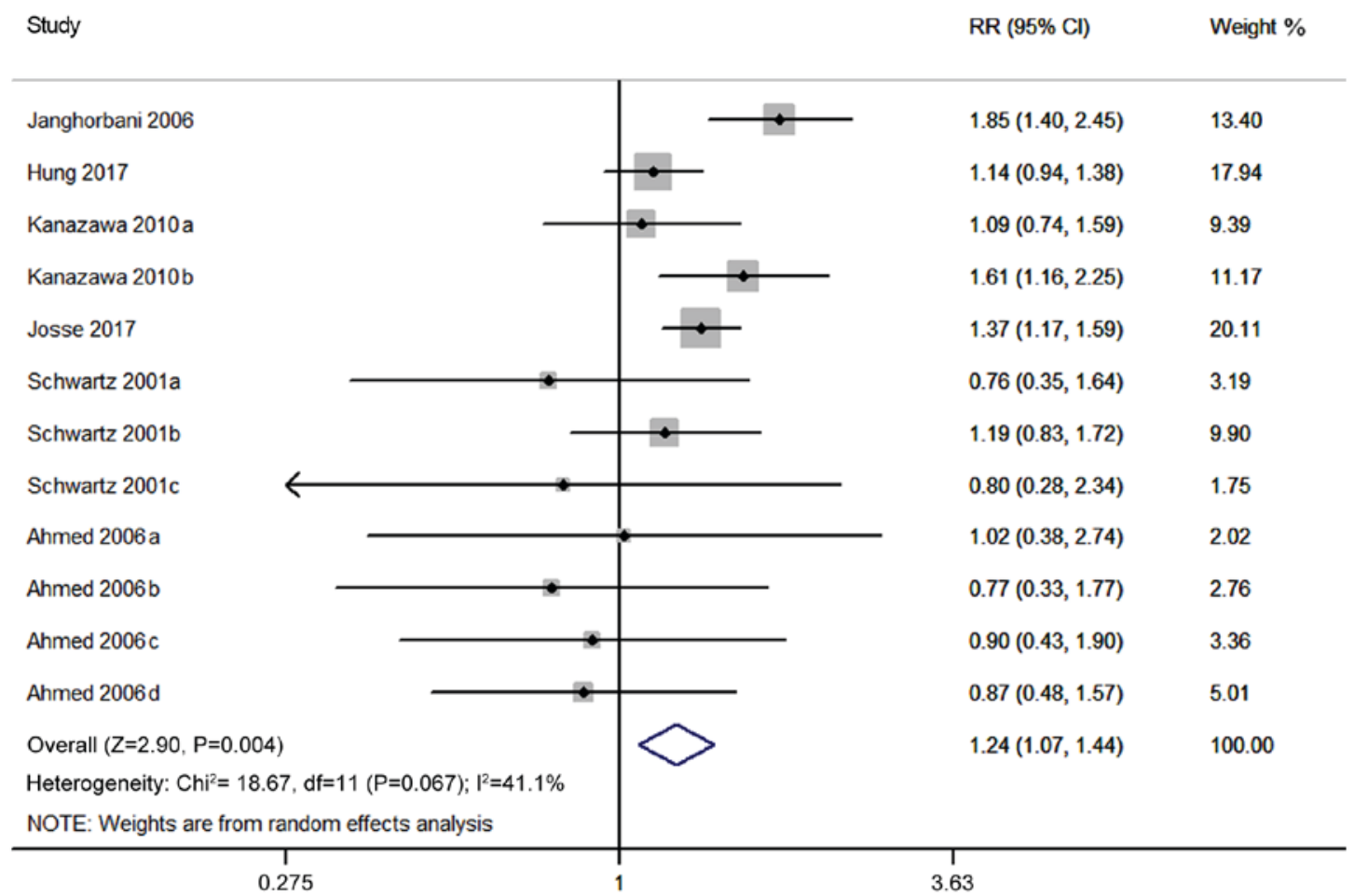

Figure 3. Sensitivity analysis verifying the robustness of the result by removing one study at a time. The risk ratio and weight for each study are presented as solid diamond and squares, respectively. The horizontal line indicates the 95\% CI. Pooled results for all studies, following exclusion of the study by Pscherer et al (13), are presented as an unfilled diamond. CI, confidence interval.

was determined by removing one study each time and re-performing the meta-analysis. Using this strategy, the study by Pscherer et al (13) was identified as the major source of heterogeneity. This may be due to the mean follow-up time being too short in the insulin and control groups when compared with the follow-up times in the other studies. 


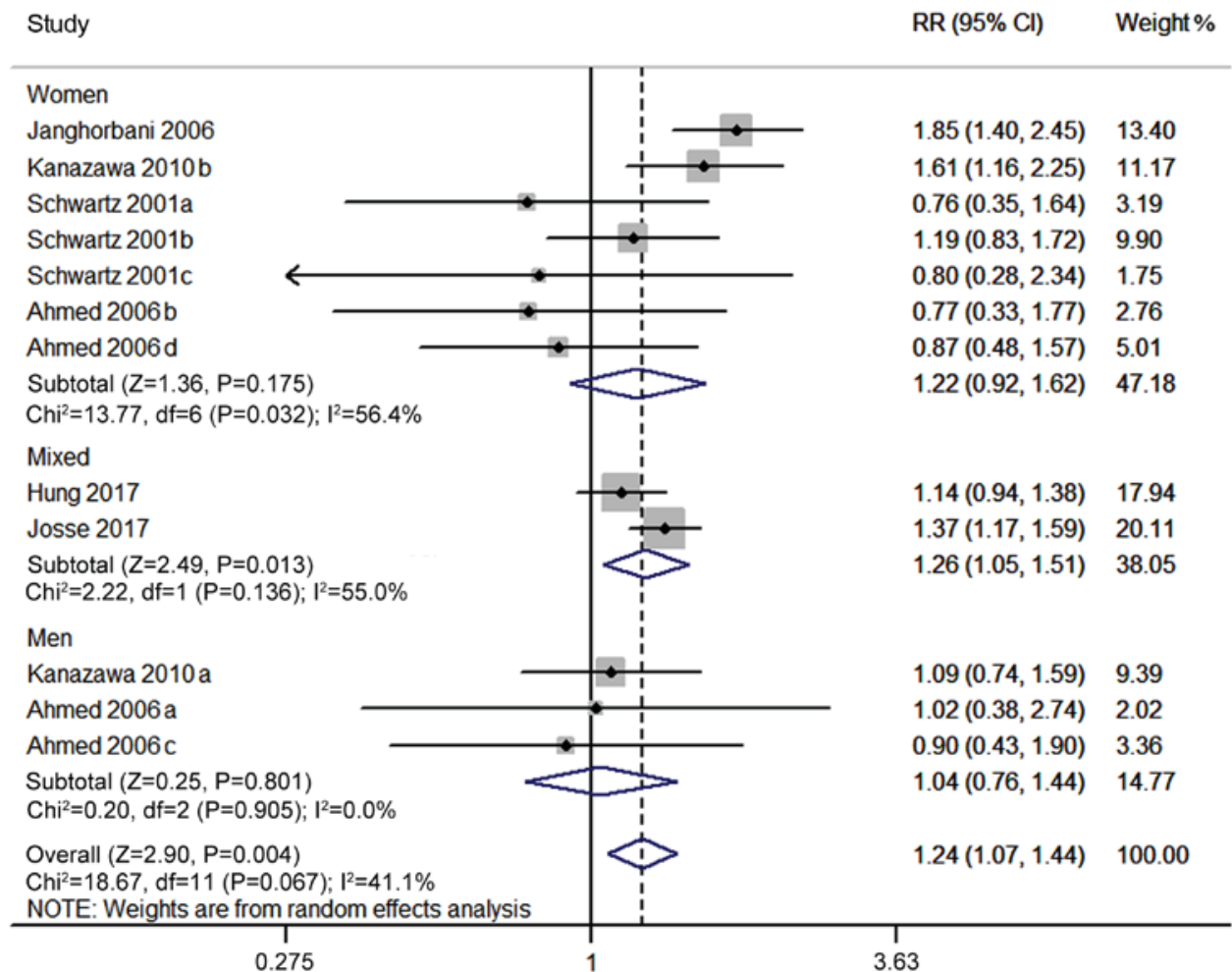

Figure 4. Subgroup analysis based on sex comparing the risk of fracture following insulin and non-insulin treatment for patients with type 2 diabetes mellitus. The risk ratio and weight for each study are presented as solid diamond and squares, respectively. The horizontal line indicates the $95 \% \mathrm{CI}$ and the dotted line indicates the pooled risk ratio. Pooled results for all studies are presented as an unfilled diamond. CI, confidence interval.

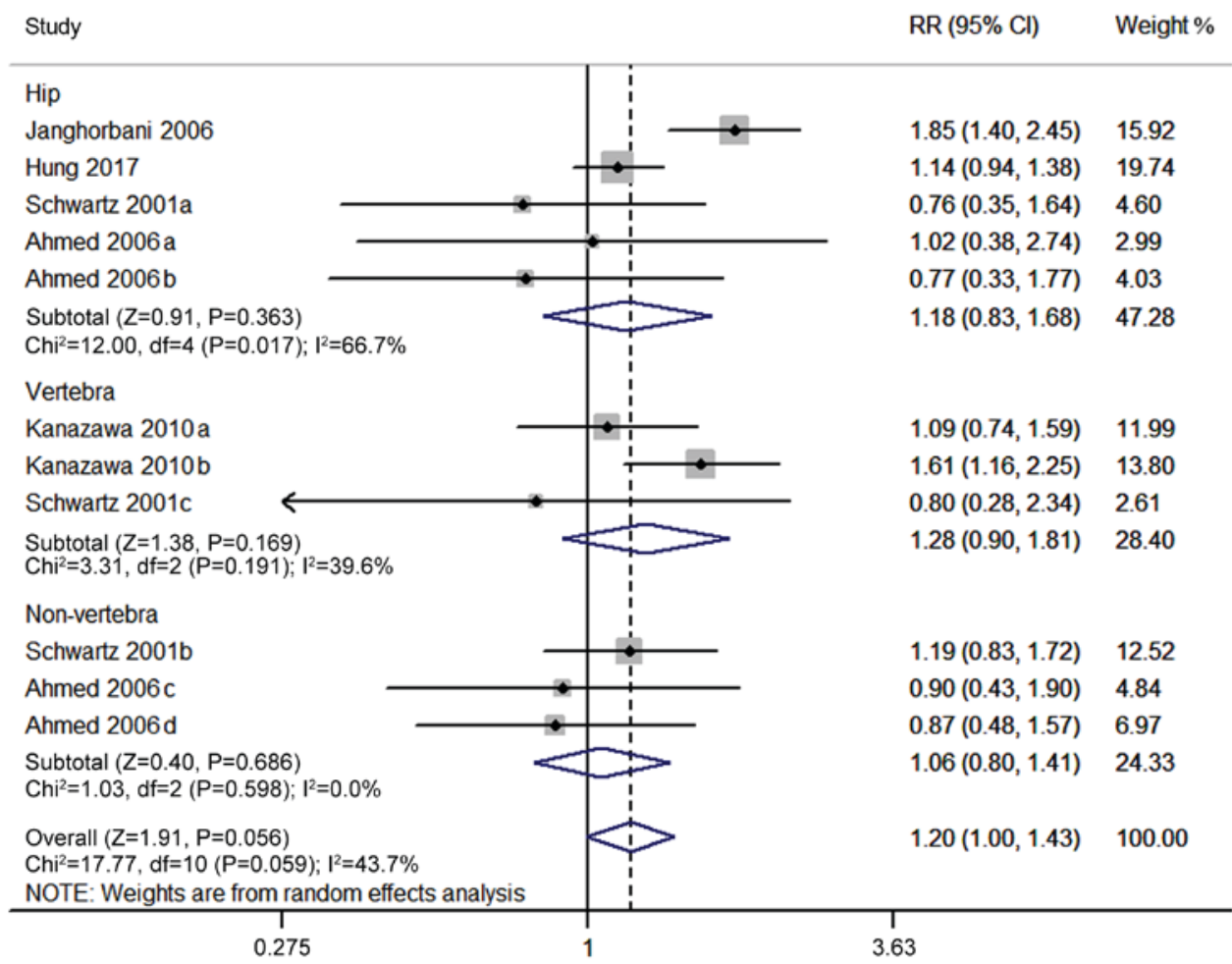

Figure 5. Subgroup analysis based on fracture site comparing the risk of fracture following insulin and non-insulin treatment for patients with type 2 diabetes mellitus. The risk ratio and weight for each study are presented as solid diamond and squares, respectively. The horizontal line indicates the $95 \% \mathrm{CI}$ and the dotted line indicates the pooled risk ratio. Pooled results for all studies are presented as an unfilled diamond. CI, confidence interval. Note: non-vertebra includes the hip.

Furthermore, the mean follow-up time was unbalanced in the insulin group (2.0 years) and the control group (2.5 years), which may have led to bias regarding in the incidence of fracture. This indicated that the inconsistent follow-up periods 


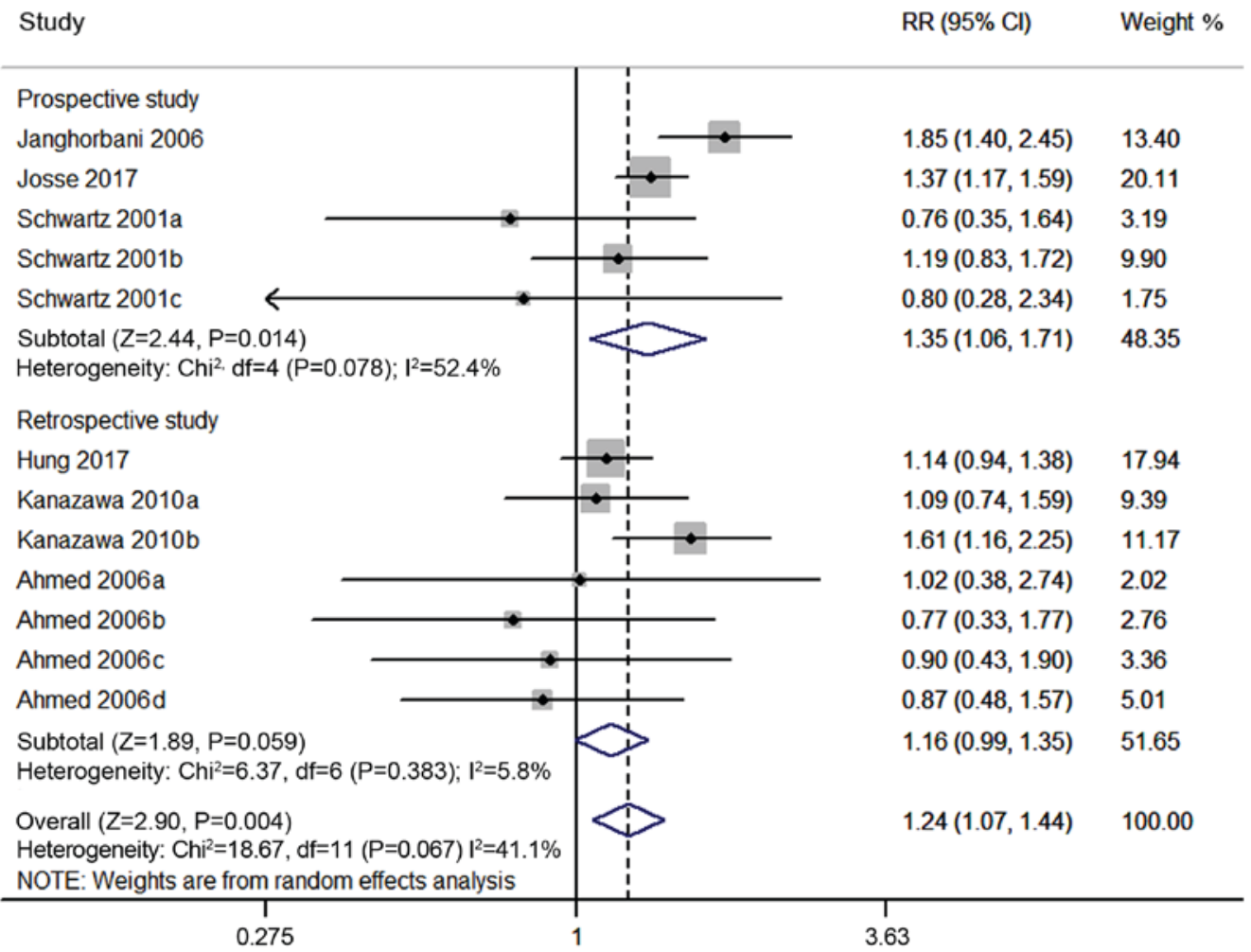

Figure 6. Subgroup analysis based on study design comparing the risk of fracture following insulin and non-insulin treatment for patients with type 2 diabetes mellitus. The risk ratio and weight for each study are presented as solid diamond and squares, respectively. The horizontal line indicates the $95 \% \mathrm{CI}$ and the dotted line indicates the pooled risk ratio. Pooled results for all studies are presented as an unfilled diamond. CI, confidence interval.

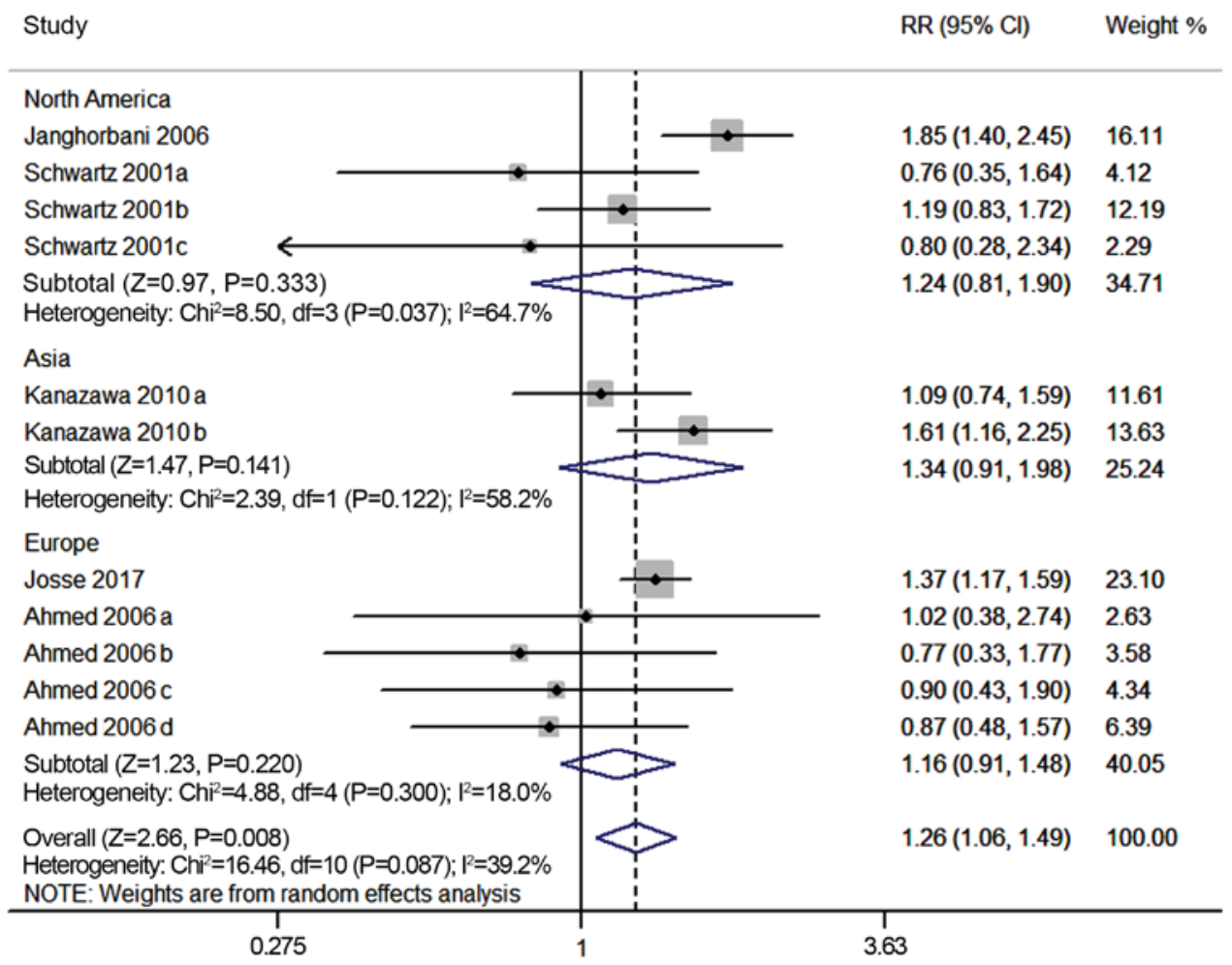

Figure 7. Subgroup analysis based on region comparing the risk of fracture following insulin and non-insulin treatment for patients with type 2 diabetes mellitus. The risk ratio and weight for each study are presented as solid diamond and squares, respectively. The horizontal line indicates the $95 \%$ CI and the dotted line indicates the pooled risk ratio. Pooled results for all studies are presented as an unfilled diamond. CI, confidence interval.

may account for the heterogeneity observed. In addition, the heterogeneity also changed after subgroup analysis. Some subgroups had very low heterogeneity while some subgroups still remained at moderate levels of heterogeneity. Therefore, 
it may be postulated that sex, fracture site, study design and region may have contributed to the heterogeneity in the present meta-analysis.

Insulin, as an anabolic agent in the bone, stimulates the regulation of osteoblast function, increasing the proliferation and differentiation from mesenchymal stem cells (17). In a study using a mouse model, insulin receptor substrates caused osteopenia with decreased bone formation and increased bone resorption (18). According to a clinical study, osteopenia and osteoporosis are frequent complications in adult and pediatric patients with T1DM (6). In a 7-year follow-up study in young patients (age range, 20-36 years) with T1DM, intensive insulin therapy stabilized the BMD and decreased bone resorption markers (19). In T2DM, insulin resistance reduces the efficiency of insulin in promoting glucose uptake and utilization, leading to relative insulin deficiency. The body produces compensatory amounts of insulin to maintain stable blood glucose levels, resulting in hyperinsulinemia. Furthermore, it is well-known that once insulin therapy is established, adequate levels of portal insulin are obtained at the expense of peripheral hyperinsulinemia, which may have an impact on various organs and tissues. Certain studies have indicated that insulin resistance, particularly hyperinsulinemia, may negatively affect bone strength relative to load $(20,21)$.

The mechanisms responsible for the increased fracture risk in insulin users remain to be fully elucidated. There are several potential mechanisms. First, most studies have reported that endogenous insulin is an anabolic agent in bone (22-24), whereas the effect of exogenous insulin remains ambiguous (25-31). Barrett-Connor and Kritz-Silverstein (32) reported that exogenous insulin therapy removes the impact of endogenous insulin as an anabolic agent on bone. Second, diabetic insulin users are usually more likely to have chronic hyperglycemia, which may impair bone quality (20). Third, patients requiring insulin administration usually have a long history of diabetes and diabetic complications, which may affect the occurrence of bone fractures. Several studies have indicated that advanced disease increases the risk of fracture $(17,33)$. Fourth, insulin treatment is associated with an increased tendency to fall during a hypoglycemic episode. Kennedy et al (12) observed that insulin-treated subjects were more likely to fall and sustain bone fracture due to a fall during a hypoglycemic episode when compared to non-insulin-treated patients. Finally, insulin users are more likely to have diabetic retinopathy and peripheral neuropathy, which increases the prevalence of chronic gait/balance impairments and impaired vision $(4,9)$.

Of note, the present study had certain limitations. First, a certain degree of heterogeneity was present in the current meta-analysis, even after removing one study. Bias analysis was also not performed due to the relatively small number of studies included in this analysis. Second, several confounding factors, including BMD, BMI, calcium/vitamin D levels, glycosylated hemoglobin, duration of diabetes, dose of insulin, level of glucose and any other treatments affecting the fracture risk, were not considered, as most of studies did not include this data and therefore the pooled adjusted RR was not calculated. Further studies are necessary to analyze the correlation of different statuses of insulin and fracture risk. Third, the present study did not include patients with T1DM, as only few studies reported on it; certain studies have indicated that different types of DM affect bone to varying degrees and through various mechanisms $(34,35)$. Fourth, the study population mostly included elderly participants and post-menopausal women who appeared to have higher insulin intake, and this may have biased the outcomes. A further meta-analysis should be performed to examine age and menopausal status of women with T1DM. The geographical distribution was another possible limitation of the present results, with only one out of seven studies were conducted in Asia. Therefore, further meta-analysis is required and should include are more studies from Asia.

Despite these limitations, the present meta-analysis indicated that insulin treatment is associated with an increased fracture risk among patients with T2DM, when compared to oral anti-diabetic drugs. Subgroup analysis indicated that the association was influenced by sex, fracture site, study design and geographical region. These results suggest that the risk of fracture associated with insulin use should be considered in the process of deciding on a treatment approach. However, further randomized, controlled studies are required to confirm these results.

\section{Acknowledgements}

Not applicable.

\section{Funding}

No funding was received.

\section{Availability of data and materials}

All datasets used and/or analyzed during the current study are available from the corresponding author on reasonable request.

\section{Authors' contributions}

YZ and QC contributed to the conception and design of the study, the analysis and interpretation of data and the revision of the manuscript. YL, LZ, YD and XM participated in literature screening and data extraction. BZ provided guidance and generated the figures. All authors read and approved the final version of the manuscript.

\section{Ethics approval and consent to participate}

Not applicable.

\section{Patient consent for publication}

Not applicable.

\section{Competing interests}

All authors declare that they have no competing interests. 


\section{References}

1. Janghorbani M, Feskanich D, Willett WC and Hu F: Prospective study of diabetes and risk of hip fracture: The Nurses' Health Study. Diabetes Care 29: 1573-1578, 2006.

2. Lipscombe LL, Jamal SA, Booth GL and Hawker GA: The risk of hip fractures in older individuals with diabetes: A population-based study. Diabetes Care 30: 835-841, 2007.

3. Schwartz AV, Sellmeyer DE, Ensrud KE, Cauley JA, Tabor HK, Schreiner PJ, Jamal SA, Black DM and Cummings SR; Study of Osteoporotic Features Research Group: Older women with diabetes have an increased risk of fracture: A prospective study. J Clin Endocrinol Metab 86: 32-38, 2001.

4. Ivers RQ, Cumming RG, Mitchell P and Peduto AJ; Blue Mountains Eye Study: Diabetes and risk of fracture: The blue mountains eye study. Diabetes Care 24: 1198-1203, 2001.

5. Russo GT, Giandalia A, Romeo EL, Nunziata M, Muscianisi M, Ruffo MC, Catalano A and Cucinotta D: Fracture risk in type 2 diabetes: Current perspectives and gender differences. Int J Endocrinol 2016: 1615735, 2016.

6. Thrailkill KM, Lumpkin CK Jr, Bunn RC, Kemp SF and Fowlkes JL: Is insulin an anabolic agent in bone? Dissecting the diabetic bone for clues. Am J Physiol Endocrinol Metab 289: E735-E745, 2005.

7. Melton LJ III, Leibson CL, Achenbach SJ, Therneau TM and Khosla S: Fracture risk in type 2 diabetes: Update of a population-based study. J Bone Miner Res 23: 1334-1342, 2008.

8. Ahmed LA, Joakimsen RM, Berntsen GK, Fønnebø V and Schirmer H: Diabetes mellitus and the risk of non-vertebral fractures: The Troms $\varnothing$ study. Osteoporos Int 17: 495-500, 2006.

9. Napoli N, Strotmeyer ES, Ensrud KE, Sellmeyer DE, Bauer DC, Hoffman AR, Dam TT, Barrett-Connor E, Palermo L, Orwoll ES, et al: Fracture risk in diabetic elderly men: The MrOS study. Diabetologia 57: 2057-2065, 2014.

10. Vestergaard P, Rejnmark L and Mosekilde L: Relative fracture risk in patients with diabetes mellitus, and the impact of insulin and oral antidiabetic medication on relative fracture risk. Diabetologia 48: 1292-1299, 2005.

11. Monami M, Cresci B, Colombini A, Pala L, Balzi D, Gori F, Chiasserini V, Marchionni N, Rotella CM and Mannucci E: Bone fractures and hypoglycemic treatment in type 2 diabetic patients: A case-control study. Diabetes Care 31: 199-203, 2008

12. Kennedy RL, Henry J, Chapman AJ, Nayar R, Grant P and Morris AD: Accidents in patients with insulin-treated diabetes: Increased risk of low-impact falls but not motor vehicle crashes-a prospective register-based study. J Trauma 52: 660-666, 2002.

13. Pscherer S, Kostev K, Dippel FW and Rathmann W: Fracture risk in patients with type 2 diabetes under different antidiabetic treatment regimens: A retrospective database analysis in primary care. Diabetes Metab Syndr Obes 9: 17-23, 2016.

14. Kanazawa I, Yamaguchi T, Yamamoto $M$ and Sugimoto T: Relationship between treatments with insulin and oral hypoglycemic agents versus the presence of vertebral fractures in type 2 diabetes mellitus. J Bone Miner Metab 28: 554-560, 2010.

15. Hung YC, Lin CC, Chen HJ, Chang MP, Huang KC, Chen YH and Chen CC: Severe hypoglycemia and hip fracture in patients with type 2 diabetes: A nationwide population-based cohort study. Osteoporos Int 28: 2053-2060, 2017.

16. Josse RG, Majumdar SR, Zheng Y, Adler A, Bethel MA, Buse JB, Green JB, Kaufman KD, Rodbard HW, Tankova T, et al: Sitagliptin and risk of fractures in type 2 diabetes: Results from the TECOS trial. Diabetes Obes Metab 19: 78-86, 2017.

17. Hamann C, Kirschner S, Gunther KP and Hofbauer LC: Bone, sweet bone-osteoporotic fractures in diabetes mellitus. Nat Rev Endocrinol 8: 297-305, 2012

18. Akune T, Ogata N, Hoshi K, Kubota N, Terauchi Y, Tobe K, Takagi H, Azuma Y, Kadowaki T, Nakamura K and Kawaguchi H: Insulin receptor substrate-2 maintains predominance of anabolic function over catabolic function of osteoblasts. J Cell Biol 159: 147-156, 2002.
19. Campos Pastor MM, López-Ibarra PJ, Escobar-Jiménez F, Serrano Pardo MD and García-Cervigón AG: Intensive insulin therapy and bone mineral density in type 1 diabetes mellitus: A prospective study. Osteoporos Int 11: 455-459, 2000.

20. Huang S, Kaw M, Harris MT, Ebraheim N, McInerney MF, Najjar SM and Lecka-Czernik B: Decreased osteoclastogenesis and high bone mass in mice with impaired insulin clearance due to liver-specific inactivation to CEACAM1. Bone 46: 1138-1145, 2010.

21. Srikanthan P, Crandall CJ, Miller-Martinez D, Seeman TE, Greendale GA, Binkley N and Karlamangla AS: Insulin resistance and bone strength: Findings from the study of midlife in the United States. J Bone Miner Res 29: 796-803, 2014.

22. Shah CS, Shah VN, Likhar N, Mothe RK, Kanukula R and Dang A: Association between bone mineral density and type 1 diabetes mellitus: A meta-analysis of observational studies. Value in Health 18: A58, 2015.

23. Klein GL: Insulin and bone: Recent developments. World J Diabetes 5: 14-16, 2014.

24. Vestergaard P: Discrepancies in bone mineral density and fracture risk in patients with type 1 and type 2 diabetes-a meta-analysis. Osteoporos Int 18: 427-444, 2007.

25. Wallander M, Axelsson KF, Nilsson AG, Lundh D and Lorentzon M: Type 2 diabetes and risk of hip fractures and non-skeletal fall injuries in the elderly: A study from the fractures and fall injuries in the elderly cohort (FRAILCO). J Bone Miner Res 32: 449-460, 2017.

26. Ghodsi M, Larijani B, Keshtkar AA, Nasli-Esfahani E, Alatab S and Mohajeri-Tehrani MR: Mechanisms involved in altered bone metabolism in diabetes: A narrative review. Diabetes Metab Disord 15: 52, 2016.

27. Adami S: Bone health in diabetes: Considerations for clinical management. Curr Med Res Opin 25: 1057-1072, 2009.

28. de Waard EAC, Koster A, Melai T, van Geel TA, Henry RMA, Schram MT, Dagnelie PC, van der Kallen CJ, Sep SJS, Stehouwer CDA, et al: The association between glucose metabolism status, diabetes severity and a history of fractures and recent falls in participants of 50 years and older-the Maastricht study. Osteoporos Int 27: 3207-3216, 2016.

29. Starup-Linde J, Gregersen S, Frost M and Vestergaard P: Use of glucose-lowering drugs and risk of fracture in patients with type 2 diabetes. Bone 95: 136-142, 2017.

30. Dutta MK, Pakhetra R and Garg MK: Evaluation of bone mineral density in type 2 diabetes mellitus patients before and after treatment. Med J Armed Forces India 68: 48-52, 2012.

31. Yan W and Li X: Impact of diabetes and its treatments on skeletal diseases. Front Med 7: 81-90, 2013.

32. Barrett-Connor E and Kritz-Silverstein D: Does hyperinsulinemia preserve bone? Diabetes Care 19: 1388-1392, 1996.

33. Napoli N, Chandran M, Pierroz DD, Abrahamsen B, Schwartz AV and Ferrari SL; IOF Bone and Diabetes Working Group: Mechanisms of diabetes mellitus-induced bone fragility. Nat Rev Endocrinol 13: 208-219, 2017

34. Oei L, Rivadeneira F, Zillikens MC and Oei EH: Diabetes, diabetic complications, and fracture risk. Curr Osteoporos Rep 13: 106-115, 2015.

35. Khan TS and Fraser LA: Type 1 diabetes and osteoporosis: From molecular pathways to bone phenotype. J Osteoporos 2015: 174186,2015

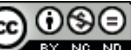

This work is licensed under a Creative Commons Attribution-NonCommercial-NoDerivatives 4.0 International (CC BY-NC-ND 4.0) License. 